\title{
Management of alcohol withdrawal: time to "SHAKE" things up?
}

\author{
Bjug Borgundvaag, PhD, MD, CCFP $(E M)^{*}+$; Michelle Klaiman, MD, FRCPC, Dip ABAM ${ }^{\ddagger \S}$
}

\section{INTRODUCTION}

More than three-quarters of Canadians over the age of 15 have consumed beverage alcohol within the last year. ${ }^{1}$ Poured at family gatherings and among friends, gifted for special occasions, and even used in religious ceremonies, alcohol is commonplace and socially acceptable in Canadian society. Usually consumed in moderation for pleasurable mood-altering effects, it is easy to overlook the negative health consequences of alcohol. The numbers, however, are sobering. Data from the Centers for Disease Control and Prevention in the United States attribute an average of 88,129 deaths/year between 2006 and 2010 to the acute and chronic consumption of alcohol. ${ }^{2}$ In Canada, more than 11 Canadian deaths/day are attributable to alcohol, as are more than half of hospitalizations for substance use disorders combined. Between 2016 and 2017, Canadians were admitted to hospital almost 80,000 times for alcoholrelated conditions, more than the total number admitted for acute coronary syndromes. ${ }^{3}$ The cost to Canadians for alcohol-related harm is significant, nearing 15 billion annually. ${ }^{4}$ The frequency of harm associated with alcohol consumption results in these patients frequently using emergency services. Despite this, emergency providers express uncertainty surrounding the best practices regarding alcohol use disorder in general, and alcohol withdrawal syndrome in particular. Alcohol withdrawal syndrome is especially relevant to emergency care providers because in its most extreme form, it can develop into life-threatening seizures and/or delirium tremens.

A 2010 Cochrane review concluded that benzodiazepines are protective against alcohol withdrawal syndrome, and have potentially protective benefits for many outcomes when compared with other drugs. ${ }^{5}$ On the basis of evidence and experience, benzodiazepines have become the drug of first choice for the treatment of alcohol withdrawal syndrome. Two randomized controlled trials have shown that compared with fixed schedule dosing, symptom triggered treatment of alcohol withdrawal syndrome, using the Clinical Institute Withdrawal Assessment (CIWA) protocol and long-acting benzodiazepines, results in much faster resolution of alcohol withdrawal syndrome symptoms, lower total doses of benzodiazepines, with no increase in adverse outcomes. ${ }^{6,7}$

The use of a symptom triggered treatment protocol for the management of alcohol withdrawal syndrome is dependent on the ability to confidently and accurately determine severity of alcohol withdrawal, and knowledge regarding the pharmacological properties of the medications used. The importance of using long-acting benzodiazepines, such as diazepam (which is slowly metabolized over days, providing protection from delirium tremens, which usually develops 3-5 days after abstention), as opposed to short half-life agents like lorazepam (which require ongoing evaluation and treatment for days), cannot be overemphasized. Even the ability to distinguish between alcohol withdrawal syndrome and alcohol craving can be challenging. These competencies are not well taught and require experience to acquire. Health care providers commonly express lack of knowledge and confidence when making clinical decisions around the management of alcohol withdrawal syndrome.

In recent years, the concept of benzodiazepineresistant alcohol withdrawal has increasingly appeared in the emergency medicine literature. ${ }^{8-10}$ In this issue of

From the *Schwartz Reisman Emergency Medicine Institute, Sinai Health System, Toronto, ON; ${ }^{\dagger}$ Department of Family Medicine, University of Toronto, Toronto, ON; ${ }^{\ddagger}$ Emergency and Addiction Medicine, St. Michael's Hospital, Toronto, ON; and the ${ }^{\S}$ Department of Medicine, University of Toronto, Toronto, ON.

Correspondence to: Dr. Bjug Borgundvaag; Mount Sinai Hospital, 206-600 University Avenue, Toronto, ON M5G 1X5, Canada; Email: Bjug.borgundvaag@sinaihealth.ca

(C) Canadian Association of Emergency Physicians

CJEM 2020;22(2):131-132

DOI 10.1017/cem.2020.7 
CFEM, Langlois et al., ${ }^{11}$ attempt to develop a consensus definition of benzodiazepine-resistant alcohol withdrawal. Given the uncertainty around the evaluation and management of alcohol withdrawal syndrome in general, it is not surprising that there was a low response rate to the survey, and that no clear preferred definition of benzodiazepineresistant alcohol withdrawal emerged. The authors found that almost two-thirds of respondents agreed with the statement that an inadequate response to a dose equivalent to $40 \mathrm{mg}$ of diazepam within the first 2 hours of treatment was indicative of benzodiazepine-resistant alcohol withdrawal. Furthermore, if the diagnosis of benzodiazepine-resistant alcohol withdrawal is made, then a change in management or addition of an adjunctive medication, such as phenobarbital, is recommended.

We applaud the authors for highlighting the importance of alcohol withdrawal syndrome and agree with their conclusion that benzodiazepine-resistant alcohol withdrawal needs to be formally defined and studied. That said, we would be concerned if readers were to accept the above definition of benzodiazepineresistant alcohol withdrawal, and its assumption of the necessity of additional medication classes. In our experience, administration of $40 \mathrm{mg}$ of diazepam in the first 2 hours of treatment is not unusual for patients with moderate or even severe alcohol withdrawal syndrome who require treatment. Adding additional medication classes to a treatment protocol that is already frequently not well understood has the potential to increase confusion and paradoxically worsen care. While barbiturates are useful and effective in the management of severe alcohol withdrawal syndrome, the safety profile of benzodiazepines in general compared with barbiturates with their narrow therapeutic index, make the former a much better option, especially outside of the intensive care unit setting (i.e., nonmonitored hallways, general medical wards) and if there is any uncertainty around dose requirements. Finally, we are concerned that defining benzodiazepineresistant alcohol withdrawal as the use of $40 \mathrm{mg}$ of diazepam in the first 2 hours of treatment will significantly increase the number of patients labeled as "severe," potentially resulting in more patients referred for admission. Inpatient management is costly, uses precious healthcare resources, and the frequency of required monitoring is difficult to provide on general medical wards.

Alcohol withdrawal syndrome is an important clinical problem frequently encountered in emergency departments in Canada and around the world. While the article by Langlois et al. is a first step in defining benzodiazepine- resistant treatment of alcohol withdrawal, we agree that more work is required to develop clear guidelines to guide treatment decisions. If we can learn to confidently identify and manage alcohol withdrawal in the emergency department, we can both improve patient care, and optimize the use of health care resources.

Keywords: Emergency medicine, toxicology, alcohol withdrawal, addiction

Competing interests: None declared.

\section{REFERENCES}

1. Government of Canada. Canadian Tobacco, Alcobol and Drugs Survey (CTADS): summary of results for 2017. Ottawa, Ontario: Government of Canada; 2017.

2. U.S. Department of Health and Human Services, Public Health Service, National Institutes of Health, National Institute on Alcohol Abuse and Alcoholism. Tenth Special Report to the US Congress on Alcohol and Health. Washington, DC: NIAAA; 2001.

3. CIHI. New Data Available on Home Care and Mental Health and Addictions: Canadian Institute for Health Information; 2019 Available at: https://www.cihi.ca/en/new-dataavailable-on-home-care-and-mental-health-and-addictions (accessed January 14, 2020).

4. Rhem J, Baliunas D, Brochu S, et al. The Costs of Substance Abuse in Canada 2002. Available at: https://www.ccsa.ca/ sites/default/files/2019-05/ccsa-011332-2006.pdf (accessed January 14, 2020).

5. Amato L, Minozzi S, Vecchi S, Davoli M. Benzodiazepines for alcohol withdrawal. Cochrane Database Syst Rev 2010(3): CD005063.

6. Saitz R, Mayo-Smith MF, Roberts MS, Redmond HA, Bernard DR, Calkins DR. Individualized treatment for alcohol withdrawal. A randomized double- blind controlled trial. 7AMA 1994;272(7):519-23.

7. Daeppen JB, Gache P, Landry U, et al. Symptom-triggered vs fixed-schedule doses of benzodiazepine for alcohol withdrawal: a randomized treatment trial. see comment. Arch Intern Med 2002;162(10):1117-21.

8. Hughes D. Benzodiazepine-Refractory Alcohol Withdrawal REBEL EM2016 Available at: https://rebelem.com/benzodi azepine-refractory-alcohol-withdrawal/ (accessed January $14,2020)$.

9. Hack JB, Hoffmann RS, Nelson LS. Resistant alcohol withdrawal: does an unexpectedly large sedative requirement identify these patients early? $\mathcal{f}$ Med Toxicol 2006;2(2):55-60.

10. Benedict NJ, Wong A, Cassidy E, et al. Predictors of resistant alcohol withdrawal (RAW): a retrospective case-control study. Drug Alcohol Depend 2018;192:303-8.

11. Langlois $\mathrm{H}$, Cormier $M$, Villeneuve E, Hoffman RS, Longo C, Gosselin S. Benzodiazepine resistant alcohol withdrawal: what is the clinicnan's preferred definition? CFEM 2020;22(2):165-169. 